\title{
Gallbladder lymphangioma: A case report and review of the literature
}

\author{
Jwa-Kyung Kim, Kyo-Sang Yoo, Joon Ho Moon, Kwang Hyuk Park, Yong Woo Chung, Kyoung Oh Kim, Cheol Hee \\ Park, Taeho Hahn, Sang Hoon Park, Jong Hyeok Kim, Jang Yeong Jeon, Min Jung Kim, Kwang Seon Min, Choong \\ Kee Park
}

Jwa-Kyung Kim, Kyo-Sang Yoo, Joon Ho Moon, Kwang Hyuk Park, Yong Woo Chung, Kyoung Oh Kim, Cheol Hee Park, Taeho Hahn, Sang Hoon Park, Jong Hyeok Kim, Choong Kee Park, Department of Gastroenterology and Hepatology, Hallym University College of Medicine, Anyang, Korea

Jang Yeong Jeon, Department of Surgery, Hallym University College of Medicine, Anyang, Korea

Min Jung Kim, Department of Diagnostic Radiology, Hallym University College of Medicine, Anyang, Korea

Kwang Seon Min, Department of Pathology, Hallym University College of Medicine, Anyang, Korea

Correspondence to: Kyo-Sang Yoo, MD, PhD, Department of Gastroenterology and Hepatology, Hallym University Sacred Heart Hospital, 896 Pyeongchon-dong, Dongan-gu, Anyang, Gyeonggi 431-070, Korea. stanyoo@hallym.ac.kr

Telephone: +82-31-380-6065 Fax: +82-31-386-2269

Received: 2006-11-08 Accepted: 2006-12-06

\begin{abstract}
Lymphangiomas are rare, benign tumors of the lymphatic system, usually present in children aged 5 years and younger. Because they are asymptomatic until the mass enlarges to cause symptoms, most lymphangiomas are diagnosed at adulthood incidentally. We experienced a case of a 60 -year-old man diagnosed with a cystic lymphangioma of the gallbladder, which was successfully resected without any complication. Magnetic resonance imaging and magnetic resonance cholangiopancreatography were very helpful for the diagnosis of the cystic lesion around the gallbladder as were ultrasonography and computed tomography scan. These showed a multi-lobulated cystic mass with intact cystic duct and bile duct in the gallbladder fossa. The patient underwent an open cholecystectomy and the histological findings were consistent with a cystic lymphangioma of the gallbladder. We here report the case of cystic lymphangioma of the gallbladder with a review of the literature.
\end{abstract}

(c) 2007 The WJG Press. All rights reserved.

Key words: Lymphangioma; Gallbladder; Cholecystectomy

Kim JK, Yoo KS, Moon JH, Park KH, Chung YW, Kim KO, Park $\mathrm{CH}$, Hahn T, Park SH, Kim JH, Jeon JY, Kim MJ, Min KS, Park CK. Gallbladder lymphangioma: A case report and review of the literature. World J Gastroenterol 2007; 13(2): 320-323 http://www.wjgnet.com/1007-9327/13/320.asp

\section{INTRODUCTION}

Lymphangiomas are uncommon benign congenital malformations of the lymphatic system ${ }^{[1-3]}$. About $95 \%$ lymphangiomas occur in the skin and the subcutaneous tissues of the head, neck and axillary region and the remaining 5\% appear in other parts of the body such as lungs, pleura, pericardium, esophagus, stomach, jejunum, colon, pancreas, liver, gallbladder, kidney, and the mesentery ${ }^{[3-5]}$. Because most lymphangiomas are multicystic, lobulated lesions, they may be confused with other lesions, such as intrahepatic simple cysts, ductal ectasia, liver hemangiomas, pericholecystic cystic tumors or angiosarcomas. Various imaging studies are often required for accurate diagnosis and to differentiate gallbladder lymphangiomas from other lesions ${ }^{[3,6,7]}$. Recently, magnetic resonance imaging (MRI) and magnetic resonance cholangiopancreatography (MRCP) have been shown to be helpful for the characterization of the lesions as well as an evaluation of the relation to the adjacent organs. Complete surgical resection is the treatment of choice for gallbladder lymphangioma, and recently successful laparoscopic resection also has been reported ${ }^{[8]}$.

\section{CASE REPORT}

A 60-year-old man was admitted to Hallym University Sacred Heart Hospital for treatment of aspiration pneumonia. He presented a high fever, chills and coughing, and had no abdominal pain or discomfort. Chest computed tomography (CT) scan for evaluation of the pneumonia unexpectedly showed a large lobulating low density lesion in the liver near the gallbladder in addition to pneumonic infiltration of the lungs. The patient was referred to our department for evaluation of the large cystic lesion. Abdominal ultrasonography (US) showed a hypoechoic mass encircling the gallbladder lumen in the liver (Figure 1). Abdominal CT showed an approximately $5.2 \mathrm{~cm}$-sized multi-lobulated cystic mass, insinuating gallbladder lymphangioma, between the liver and the gallbladder bed (Figure 2). Abdominal MRI was performed for the characterization and anatomical evaluation of the lesion. Axial T2-weighted image showed an intact lumen 


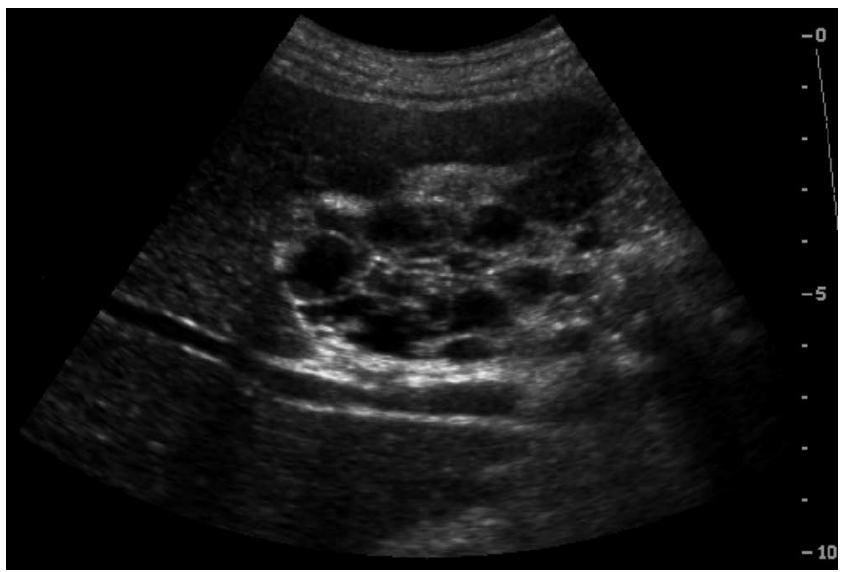

Figure 1 Abdominal ultrasonography showing multi-loculated cystic mass and intact gallbladder lumen.

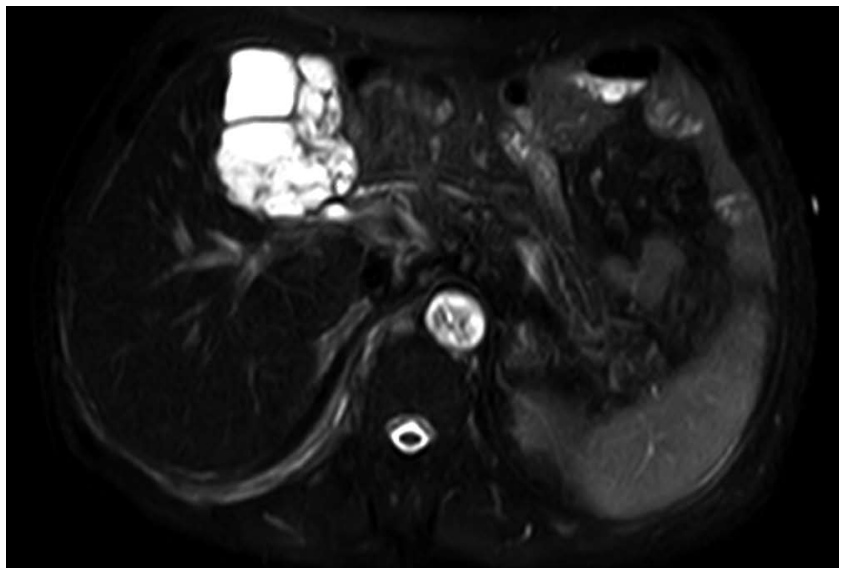

Figure 3 Axial T2-weighted magnetic resonance (MR) image showing a thin multiseptated cystic mass (high-signal intensity) with scalloping margin.

of the gallbladder (low-signal intensity) surrounded by a thin multi-septated cystic mass (high-signal intensity) with scalloping margin (Figure 3). This finding strongly suggested a lymphangioma arising from the lymphatic tissue of the gallbladder wall. MRCP also clearly showed a large multi-cystic gallbladder lymphangioma in the gallbladder fossa (Figure 4). Characteristically, the cystic duct and bile duct were completely separated from the lymphangioma, and were well preserved. Additionally, the gallbladder was obviously visualized and its contraction was grossly normal (ejection fraction $=89 \%$ ) on hepatobiliary DISIDA scan.

An open cholecystectomy was performed after resolution of the pneumonia. At surgical exploration, a well-defined multi-lobular cystic tumor encapsulating the gallbladder was observed. Grossly the tumor was measured $5.5 \mathrm{~cm} \times 4.3 \mathrm{~cm} \times 3.5 \mathrm{~cm}$ in size and was clearly separated from the liver. When the tumor was cut off from the surface, the gallbladder mucosa was intact and varioussized multiple cysts were shown. Each cyst was filled with serous or hemorrhagic lymphatic fluid. Histological evaluation revealed a flat endothelial lining encircling each cyst and multiple lymphocyte aggregations beside the cyst (Figure 5). This demonstrated that the cyst was a lymphatic

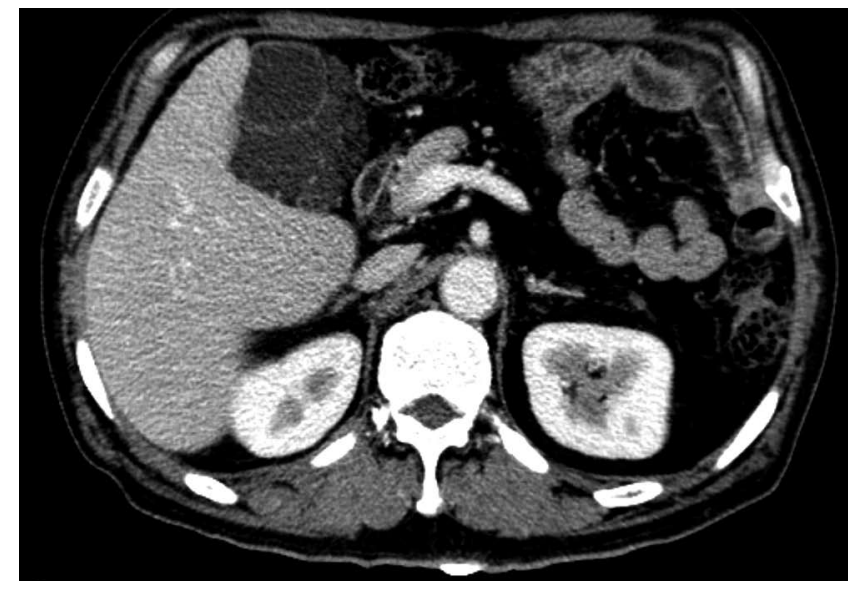

Figure 2 Abdominal computed tomography (CT) image showing a low-density multilocular cystic lesion with septation originated from the gallbladder fossa.

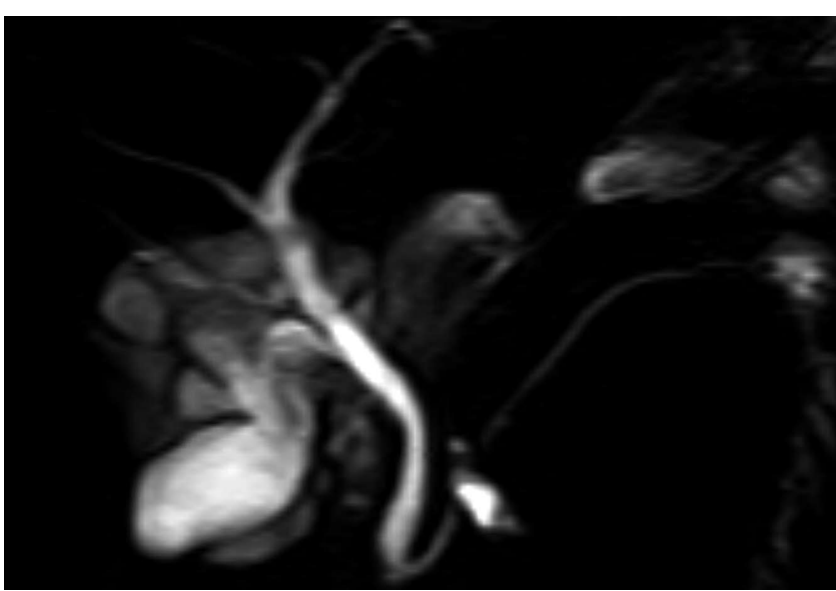

Figure 4 Magnetic resonance cholangiopancreatography (MRCP) image showing gallbladder encapsulated by variable sized multi-cystic lesions, which have no communication with biliary system.

space. The lesion was diagnosed as a cystic lymphangioma of the gallbladder because it had no connection with adjacent normal lymphatics. CT at 12 mo following the surgical resection showed no evidence of recurrence.

\section{DISCUSSION}

Lymphangiomas are benign tumors that may appear in any organ of the body except the brain. It more commonly involves the skin and soft tissue of the head and neck $(95 \%)$, and lymphangioma occurring in the gastrointestinal tract is rare. A lymphangioma arising from the gallbladder is extremely rare, representing only $0.8 \%-1 \%$ of all intraabdominal lymphangiomas ${ }^{[1-5]}$, and only a few cases have been reported. Lymphangiomas are classified as simple, cavernous, and cystic types based on their histological findings ${ }^{[3,6,9-11]}$. The simple type is usually situated superficially in the skin and is composed of small thinwalled lymphatic vessels. The cavernous type is composed of dilated lymphatic vessels and lymphoid stroma, and has a connection with spaces of various normal adjacent lymphatics. The cystic type consists of lymphatic spaces 

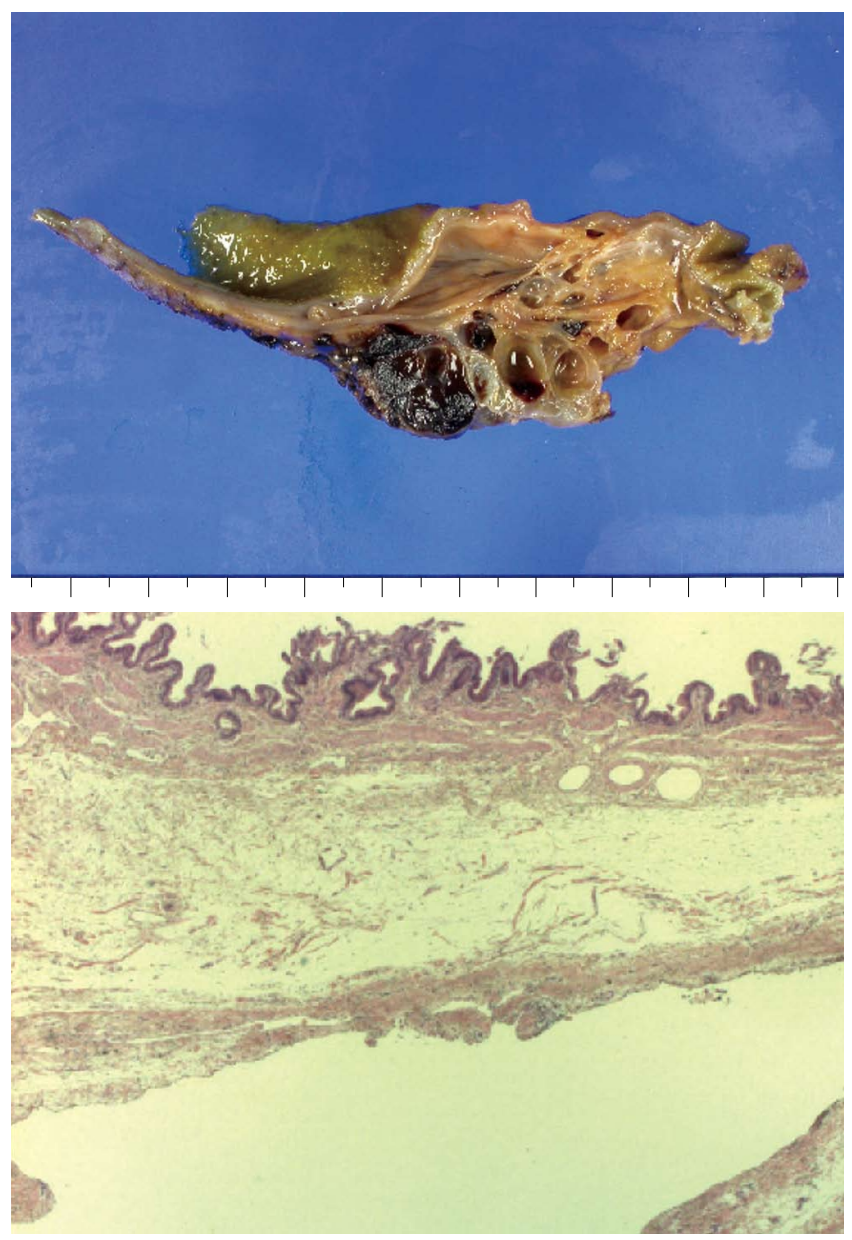

Figure 5 Gross appearance of resected specimen showing a well-marginated multi cystic lesion filled with yellowish fluid, and microphotograph from the cholecystectomy specimen showing a lymphatic space lined with flat endothelium (hematoxylin and eosin staining, $\times 12.5$ ).

of various sizes that contain fascicles of smooth muscle and collagen bundles, but has no connection with adjacent normal lymphatics ${ }^{[11,12]}$.

The etiology of lymphangiomas could be explained by several theories ${ }^{[1,6]}$. The most powerful one is that it is a congenital abnormality of the lymphatic system. Failure to establish connection with the normal drainage vessel causes sequestration of lymphatic tissue during embryogenesis. This theory would explain why lymphangioma occurs primarily in children. However, other views suggest that secondary causes such as abdominal trauma, lymphatic obstruction, inflammatory processes, surgery, or radiation therapy may lead to the formation of lymphangiomas ${ }^{[1,6]}$.

Clinical presentations of these tumors are variable. Most cases are asymptomatic or have non-specific symptoms, such as nausea, vomiting, dyspepsia, abdominal discomfort or a palpable mass. In the past it was somewhat difficult to diagnose a lymphangioma before surgical or histological identification. Preoperative diagnosis of gallbladder lymphangioma remains difficult because it is very rare, and sometimes imaging studies such as US and CT cannot distinguish it from other lesions. The findings of US and CT show a large cystic lesion, but it is difficult to find where the lesion originates from and to understand its relation to surrounding organs.
However recently, various advanced imaging studies, especially MRI and MRCP, can support the diagnosis of lymphangiomas more easily before exploration ${ }^{[1,6,7]}$. US seems to be useful in defining the mass as a multilocular cystic lesion, but often does not distinguish between a hepatic lesion and a pericholecystic lesion. CT shows a simple or multilocular cystic lesion with the density of water. The septa of the cysts are of uniform thickness. Administering contrast agents intravenously can enhance the wall of the cysts ${ }^{[6]}$. However, the findings of CT of previous reports only showed cystic lesion in the gallbladder fossa or inferior to the liver, but could not show that the lesion was arising from the gallbladder. Moreover, the gallbladder was not visible due to cystic lesions in most of them, as was evident in the present case. Although hemorrhage within the lymphangioma was readily noted on follow-up CT, it might have been difficult to differentiate it from an intracystic solid lesion if the case was initially presented after hemorrhage ${ }^{[6]}$.

In our study, MRI and MRCP are very helpful for making the correct diagnosis. One recent report described the MR findings of gallbladder lymphangiomas based on the findings of splenic cystic lymphangiomas ${ }^{[6]}$. This report suggested that characteristic MR findings would be very helpful for the differential diagnosis of gallbladder lymphangioma, and characterizing the mass even if the cystic lesion was complicated by intracystic hemorrhage. Axial T1- and T2-weighted and coronal MR images clearly depicted the lumen of the gallbladder and the multiseptated cystic mass originating in the gallbladder wall. MR imaging, including MRCP, clearly defined the mass and its relation to the gallbladder. The characteristic thin-walled multilocular cystic appearance was clearly depicted on MR imaging and was helpful for making the correct diagnosis ${ }^{[1,3,6,7]}$. MRCP characteristically showed that the cystic duct and bile duct were well preserved and were completely separated from the lymphangioma, as the finding of the present case. This was the typical feature of such lymphangiomas that had no communication between the mass and the biliary system. Therefore, if a gallbladder lymphangioma is suspected, MRI and MRCP should be recommended.

For the management of lymphangiomas, complete total surgical excision is known to be the standard treatment. If the mass grows large to compress surrounding structures and vessels, it can cause significant symptoms and morbidities. Furthermore, if it progresses to be complicated, intra-abdominal infection, rupture, torsion or hemorrhage can occur. A recent report suggested that two cases of gallbladder lymphangiomas were successfully treated by laparoscopic cholecystectomy, even though their size was more than $15 \mathrm{~cm}$ in diameter ${ }^{[8]}$. While we did not perform laparoscopic cholecystectomy, laparoscopic resection was possible because the lesion was relatively small compared to the previous report and was easily separated from the adjacent organs at surgical exploration. However, in a recent case, en bloc resection of the extrahepatic bile duct including the gallbladder was necessary because a huge cystic lesion adhered to the entire extrahepatic bile duct anteriorly and posteriorly, and part of the cyst also adhered to the right hepatic $\operatorname{artery}^{[1]}$. Therefore, laparoscopic resection was preferably 
performed after evaluation of the extent of the cystic mass and its relation to adjacent organs. Recurrence has been reported with incomplete resection, but if the lesion is completely resected, long-term prognosis is excellent ${ }^{[12]}$.

\section{REFERENCES}

1 Noh KW, Bouras EP, Bridges MD, Nakhleh RE, Nguyen JH. Gallbladder lymphangioma: a case report and review of the literature. J Hepatobiliary Pancreat Surg 2005; 12: 405-408

2 Ohba K, Sugauchi F, Orito E, Suzuki K, Ohno T, Mizoguchi N, Koide T, Terashima H, Nakano T, Mizokami M. Cystic lymphangioma of the gall-bladder: a case report. J Gastroenterol Hepatol 1995; 10: 693-696

3 Lörken M, Marnitz U, Manegold E, Schumpelick V. Intraabdominal lymphangioma. Chirurg 2001; 72: 72-77

4 Bishop MD, Steer M. Pancreatic cystic lymphangioma in an adult. Pancreas 2001; 22: 101-102

5 Takiff $\mathbf{H}$, Calabria R, Yin L, Stabile BE. Mesenteric cysts and intra-abdominal cystic lymphangiomas. Arch Surg 1985; 120: 1266-1269
6 Choi JY, Kim MJ, Chung JJ, Park SI, Lee JT, Yoo HS, Kim L, Choi JS. Gallbladder lymphangioma: MR findings. Abdom Imaging 2002; 27: 54-57

7 Vargas-Serrano B, Alegre-Bernal N, Cortina-Moreno B, Rodriguez-Romero R, Sanchez-Ortega F. Abdominal cystic lymphangiomas: US and CT findings. Eur J Radiol 1995; 19: 183-187

8 Yang HR, Jan YY, Huang SF, Yeh TS, Tseng JH, Chen MF. Laparoscopic cholecystectomy for gallbladder lymphangiomas. Surg Endosc 2003; 17: 1676

9 Yoshida Y, Okamura T, Ezaki T, Yano K, Kodate M, Murata I, Kaido M. Lymphangioma of the oesophagus: a case report an $\mathrm{d}$ review of the literature. Thorax 1994; 49: 1267-1268

10 Roisman I, Manny J, Fields S, Shiloni E. Intra-abdominal lymphangioma. Br J Surg 1989; 76: 485-489

11 Chung JH, Suh YL, Park IA, Jang JJ, Chi JG, Kim YI, Kim WH A pathologic study of abdominal lymphangiomas. J Korean Med Sci 1999; 14: 257-262

12 Amadori G, Micciolo R, Poletti A. A case of intra-abdominal multiple lymphangiomas in an adult in whom the immunological evaluation supported the diagnosis. Eur J Gastroenterol Hepatol 1999; 11: 347-351

S- Editor Liu Y L- Editor Zhu LH E- Editor Liu WF 